\title{
CRITICAL POINTS OF HARMONIC FUNCTIONS ON DOMAINS IN $R^{3}$
}

BY

\author{
ROBERT SHELTON
}

\begin{abstract}
It is shown that the critical point relations of Morse theory, together with the maximum principle, comprise a complete set of critical point relations for harmonic functions of three variables. The proof proceeds by first constructing a simplified example and then developing techniques to modify this example to realize all admissible possibilities. Techniques used differ substantially from those used by Morse in his solution of the analogous two-variable problem.
\end{abstract}

Introduction. Critical point theory is a subject which has been of pure and applied interest for many years. Morse resolved the problem associated with the study of critical points of nondegenerate functions of several variables in the late 1920's. A. Sard, a student of Morse, is credited with the discovery of a method to perturb a function with a degenerate critical point and to split or bifurcate it into several nondegenerate critical points. $R$. Thom has done famous recent work on applications of bifurcation theory to many difficult problems in biology as well as other areas not usually associated with differential topology.

This paper deals with an aspect of the following classical problem. A certain collection of functions is fixed and we seek a full description of the critical points theory of the functions considered as a class. If the functions are merely $C^{\infty}$, then the best available description of their critical point theory is contained in the Morse inequalities [3]. These inequalities relate the numbers and the kinds of critical points of a function with the Betti numbers of the domain. In his book, Topological methods in the study of functions of a complex variable [1], Morse did a complete treatment of the critical points theory of functions of two variables which resemble harmonic functions topologically. In doing this, he proved all the standard theorems relating zeros, poles, and branch points of his class of pseudoharmonic and pseudomeromorphic functions, the latter of which he called inner transformations. It would seem that the critical point behavior of harmonic functions of two variables should then be determined solely by topological considerations. In this vein, Morse proved a completeness result which can be summarized as follows. Given integers which satisfy the Morse inequalities for a harmonic function on a topological disc (including the condition that no interior point can be an extremum), there exists a region $\Omega$ in $R^{2}, \Omega$ homeomorphic to a two-cell, and a function $U$ such that the given integers describe the critical points of $U$ on $\Omega$ and its boundary. Roughly speaking, it means that the theory of critical points of

Received by the editors October 3, 1978 and, in revised form, June 28, 1979 and December 26, 1979. AMS (MOS) subject classifications (1970). Primary 58E05.

(C) 1980 American Mathematical Society $0002-9947 / 80 / 0000-0407 / \$ 06.50$ 
harmonic functions of two variables is as general as the theory for the differentiable functions which obey the maximum principle. If it were not known directly, this result would imply the accessibility of the theorems of complex analysis by topological methods.

The present work is a direct generalization of [2] to harmonic functions of three variables. It is a negative result which may be summarized as follows. Any extension of the critical point theory of harmonic functions in the plane to harmonic functions of three variables must describe behavior permitted by the maximum principle and the topological considerations which apply to the class of differentiable functions. These constraints are so weak as to essentially preclude any meaningful extensions of the theorems in [1].

As in [2], harmonic functions are constructed with critical points prescribed in advance. Morse called such functions, together with their domains, harmonic realizations of type numbers (type numbers are integer solutions of the Morse inequalities). We have retained this terminology. The constructive techniques of this work differ radically from those used in [2]. The functions in Morse's two-dimensional examples are essentially homogeneous harmonic polynomials, whereas the functions used in this paper are the potentials of charge distributions. These distributions are composed of finite unions of smooth, compact subsets of $R^{3}$, each having codimension two or more. The geometric character of these sets is closely related to the type numbers which are to be realized.

We assert that it is also possible to construct harmonic realizations in the plane using the techniques of this paper. The functions in such a construction are finite sums of logarithmic singularities. For example, let $\lambda_{1}, \lambda_{2}, \ldots, \lambda_{M_{1}}$ be points in the open right half-plane. Define

$$
u_{\varepsilon}(z)=\operatorname{Re}(z)+\sum_{i=1}^{M_{1}} \varepsilon \ln \left|z-\left(1+e^{-1 / e}\right) \frac{\lambda_{i}}{\left|\lambda_{i}\right|}\right|,
$$

$|z| \leqslant 1$. Then, for $\varepsilon$ sufficiently small and positive, the function $u_{e}(z)$ on the unit disc is a harmonic realization of the basic set of type numbers $\mu_{0}=M_{1}+1$, $\mu_{1}=0$. To realize any set of type numbers, we need only modify the boundary of the domain as in Theorem 4.2 of [2]. To verify these assertions it is necessary to use arguments similar to those of Theorems 3 and 4.

An intriguing but far more difficult problem is the following. Is it possible to construct harmonic realizations in three dimensions using polynomials as in [2]? The examples of this paper indicate that there are no analytical obstructions to doing this. Moreover, a careful study of these examples is a natural starting point in the search for the more natural polynomial examples.

1.0. Preliminaries. Let $u$ be a function of class $C^{2}$ defined in some neighborhood of the origin in $R^{n}$. The function, $u$, will be called nondegenerate or n.d. if gradient of $u$ at the point $X$ equals zero, written $\operatorname{grad}(u(X))=0$, implies matrix of second derivatives of $u$ at $X$, written $H u(X)$, is nonsingular. If $P$ is a critical point of $u$, i.e. $\operatorname{grad}(u(P))=0$, and $u$ is n.d. at $P$, then the index of the critical point, $P$, is defined to be the number of eigenvalues of $H u(P)$ which are less than 0 . 
If $u$ is a function defined in a neighborhood of $\bar{\Omega}, \Omega$ a bounded open subset of $R^{n}$ whose boundary $\Sigma$ is an $n-1$ manifold of class $C^{2}, u$ is n.d. on $\Omega$ and $\operatorname{grad}(u)$ does not vanish on $\Sigma$, then $u$ has finitely many critical points on $\Omega$. For $i=0$, $1, \ldots, n$, the interior type numbers $M_{i}$ of $u$ on $\Omega$ are defined by $M_{i}=$ number of critical points of $u$ on $\Omega$ of index $i$.

Let $N$ be the normal to $\Sigma$ directed out of $\Omega$. Points of $\Sigma$ are termed entrant if $\operatorname{grad}(u) \cdot N<0$ and emergent if $\operatorname{grad}(u) \cdot N>0$. Let $\Sigma^{-}=$the entrant points of $\Sigma$, an open submanifold of $\Sigma$. Suppose the restriction of $u$ to $\Sigma$ is n.d. Then the restriction of $u$ to $\Sigma^{-}$has finitely many critical points, and the boundary type numbers of $u$ on $\Omega, \mu_{i}, i=0,1, \ldots, n-1$, are defined as follows: $\mu_{i}=$ the number of critical points of the restriction of $u$ to $\Sigma^{-}$which are of index $i$.

Suppose $\Omega$ is a bounded open subset of $R^{3}$ with a boundary, $\Sigma$, a 2-manifold of class $C^{2}$. If $u$ is a harmonic function defined in a neighborhood of $\bar{\Omega}$, n.d. on $\Omega$, has no critical points on $\Sigma$, and restriction of $u$ to $\Sigma$ is n.d., then the betti numbers of $\Omega$, $R_{i}, i=0,1,2$, and the type numbers of $u,\left(\mu_{i}, M_{j}\right), i=0,1,2, j=0,1,2,3$, satisfy the following relations.

(1)

$$
\begin{aligned}
& \text { a. } M_{0}=M_{3}=0 . \\
& \text { b. } \mu_{0} \geqslant R_{0} . \\
& \text { c. } \mu_{0}-\mu_{1} \leqslant R_{0}-R_{1}+M_{1} . \\
& \text { d. } \mu_{0}-\mu_{1}+\mu_{2}=R_{0}-R_{1}+R_{2}+M_{1}-M_{2} .
\end{aligned}
$$

(1) follows from Theorem 1.2 of [2]. We term a set of numbers, $\left(R_{i}, \mu_{i}, M_{j}\right)$, $i=0,1,2, j=1,2$, which satisfy (1), b, c, d, condition numbers.

If $\left(R_{i}, \mu_{i}, M_{j}\right)$ are condition numbers and are the betti numbers of a domain $\Omega$ in $R^{3}$, and the boundary and interior type numbers of a harmonic function $u$ on $\Omega$, where $u$ and $\Omega$ satisfy the hypothesis of Theorem 1.2 of [2], then the numbers are said to be harmonically realizable and the domain, $\Omega$, and function, $u$, are said to be a harmonic realization of these numbers.

In this paper, examples will be constructed to prove that all condition numbers are harmonically realizable. The harmonic functions of these examples will be essentially the electrostatic potentials of charged arcs. Theorem 1 is a statement of classical analysis which describes the local behavior of such potentials. Although Theorem 1 is proved in a more general setting, we shall only need the special case when the Source $(\sigma)$ of the potential is an arc.

1.1. Notation for Theorem 1. Unless otherwise specified, capital letters will denote points in $R^{n}(n \geqslant 3)$ or a vector-valued function. Let $\sigma$ be a compact, $d$-dimensional, $C^{\infty}$ submanifold of $R^{n}$, possibly with a boundary. For $\alpha$ an $n$-tuple of nonnegative integers, $\alpha_{1}, \alpha_{2}, \ldots, \alpha_{n}$ and $f$ a suitably differentiable function of $n$ variables, let $f_{\alpha}$ be $\partial^{m} f / \partial x_{1}^{\alpha_{1}} \partial x_{2}^{\alpha_{2}} \ldots \partial x_{n}^{\alpha_{n}}$ where $m=\sum_{k=1}^{n} \alpha_{k}$. Define the function $g$ from $R^{n}-\{0\}$ to $R_{+}$by $g(X)=|X|^{2-n}$. For each $X \in R^{n}-\sigma$, define the function $u_{\sigma, \alpha}$ by $u_{\sigma, \alpha}(X)=\int_{\sigma} g_{\alpha}(X-Z) d Z$.

Let $\sigma_{1}=$ interior of $\sigma$ and $\sigma_{0}$ be any compact subset of $\sigma_{1}$. For each $\delta>0$, let 
$T_{\delta}=\left\{X \mid X \in R^{n}, \operatorname{dist}\left(X, \sigma_{0}\right) \leqslant \delta\right\}$ and $T_{\delta}^{*}=T_{\delta}-\sigma$. Let $Z_{X}=\{Z|Z \in \sigma| Z-$, $X \mid$ is minimum $\}$. Select $\delta_{0}>0$ so that for $X \in T_{\delta_{0}}$, all the sets $\left\{Z_{X}\right\}$ are singletons, and the function defined by $Z(X)=Z_{X}$ is a $C^{\infty}$ mapping of $T_{\delta_{0}}$ into $\sigma_{1}$. For $Z \in \sigma_{1}$, let $P_{Z}$ denote the tangent plane to $\sigma_{1}$ at $Z$. Let $Y: T_{\delta_{0}} \rightarrow R^{n}, y: T_{\delta_{0}} \rightarrow R$ be functions defined by $Y(X)=X-Z(X)$ and $y(X)=|Y(X)|=$ distance from $X$ to $\sigma_{1}$.

Suppose $k=n-d \geqslant 2$. Define the function $v_{\sigma, \alpha}: T_{\delta_{0}}^{*} \rightarrow R$ by the following equations

$$
v_{\sigma, \alpha}(X)=\int_{P_{Z(X)}} g_{\alpha}(X-\lambda) d \lambda, \text { for } k+m>3, m=\sum_{k=1}^{n} \alpha_{k}
$$

and

$$
v_{o, \alpha}(X)=-\omega_{d-1} \ln y(X), \quad \text { for } k+m=2, m=\sum_{k=1}^{n} \alpha_{k}
$$

where $\omega_{d-1}$ is the area of $S^{d-1}$, the unit sphere in $R^{d}$. We take $\omega_{0}=2$. Let $\phi$ : $R^{n}-\{0\} \rightarrow R$ be the function defined by $\phi(X)=C_{d, k}|X|^{2-k}$ for $k>2$ and $\phi(X)=-\omega_{d-1} \ln |X|$ for $k=2$, where $C_{d, k}=\omega_{d-1} \int_{0}^{\pi / 2} \sin ^{d-1} \theta \cos ^{k-3} \theta d \theta$. Fix a point $X_{0}$ in $R^{n}$. Define the transformation $Y_{0}(X)=Y\left(X_{0}\right)+Y_{X_{0}}^{\prime} \cdot\left(X-X_{0}\right)$ where $Y_{X_{0}}^{\prime}$ is the Jacobian matrix of $Y$ at $X_{0}$ and - indicates matrix multiplication. Then

$$
v_{\sigma, \alpha}\left(X_{0}\right)=\left(\phi\left(\left|Y_{0}(X)\right|\right)\right)_{\alpha}
$$

where the derivatives on the right-hand side of (4) are with respect to $X$ and are evaluated at $X=X_{0}$. Since the transformations $Y(X)$ and $Y_{0}(X)$ differ by $O\left(\left|X-X_{0}\right|^{2}\right)$, we have

$$
v_{\sigma, \alpha}(X)=\left\{\begin{array}{l}
(\phi(|Y(X)|))_{\alpha}+O\left(|Y(X)|^{3-k-m}\right), \quad m+k-3>0 \\
(\phi(|Y(X)|))_{\alpha}+O(\ln |Y(X)|), \quad m+k-3=0
\end{array}\right.
$$

THEOREM 1. For every $\varepsilon>0$, there exists $a \delta>0$ such that for all $X$ in $T_{\delta}^{*}$,

$$
[y(X)]^{k+m-2}\left|u_{\sigma, \alpha}(X)-v_{\sigma, \alpha}(X)\right|<\varepsilon, \quad k+m>3,
$$

and

$$
|\ln y(X)|^{-1}\left|u_{\sigma, \alpha}(X)-v_{\sigma, \alpha}(X)\right|<\varepsilon, \quad k=2, m=0 .
$$

Proof. We prove the two cases separately.

CASE $1(m+k \geqslant 3)$. For $X \in R^{n}, Z \in \sigma_{1}, X \neq Z$, define the functions $T, V$ and $\theta$ as follows. $T(X, Z)=\tau$, where $\tau$ is the point in $P_{Z}$ closest to $X, V(X, Z)=$ $Z-T(X, Z)$ and $\theta(X, Z)=|V(X, Z)| /|X-Z|$. The situation is illustrated in Figure 1 for $n=3$ and $\sigma$ a plane curve. Note that the functions $T, V$ and $\theta$ are $C^{\infty}$. Since $\sigma_{1}$ is a smooth submanifold, $\lim \theta(X, Z)=1$ as $X$ approaches $\sigma_{1}$ through $T_{\delta_{0}}$, $Z$ in $\sigma_{1},|X-Z| \rightarrow 0$ and $\sqrt{y(X)}<|X-Z|$. Since $\sigma_{0}$ is compact, we can find positive numbers $\delta_{1}, \rho_{1}$ such that for $X$ in $T_{\delta_{1}}, Z$ in $\sigma_{1}$ and $\sqrt{y(X)}<|X-Z|<$ $\rho_{1}, \theta(X, Z)>\frac{1}{2}$. For $X \in T_{\delta_{0}}$ and $y(X)<r<\rho_{1}$, let $S_{r, X}=\{Z|Z \in \sigma| X-Z \mid,=$ $r$ \}. Note that for $r$ and $y(X)$ sufficiently small the set $S_{r, X}$ approximates a 


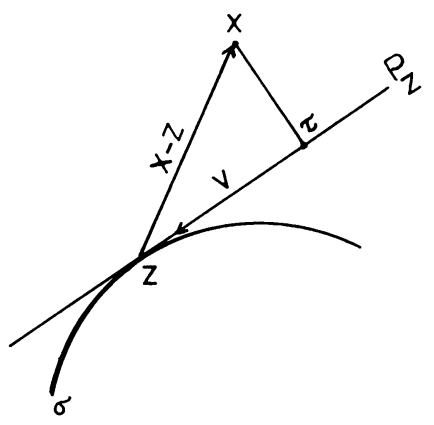

Figure 1

$(d-1)$-sphere of radius approximately equal to $\sqrt{r^{2}-y^{2}(X)}$. Let $s(r, X)=$ $\int_{S_{r, X}} d \zeta$. Since $\sigma$ is a smooth submanifold in $R^{n}, \lim \left[\omega_{d-1} r^{d-1}\right]^{-1} s(r, X)=1$ as $X \rightarrow \sigma_{1}$ through $T_{\delta_{1}}, r \rightarrow 0^{+}$, and $\sqrt{y(X)}<r$. Again by compactness of $\sigma_{0}$, we find positive numbers $\delta_{2}, \rho_{2}$ with $\delta_{2}<\delta_{1}$ and $\rho_{2}<\rho_{1}$, such that $X \in T_{\delta_{2}}$ and $\sqrt{y(X)}<r<\delta_{2}$ implies $s(r, X)<2 \omega_{d-1} r^{d-1}$. We also insist that $\delta_{2}, \rho_{2}$ be so small that whenever $X \in T_{\delta_{2}}$ and $0<r<\rho_{2}, S_{r, X}$ does not intersect the boundary of $\sigma$. To save notation, let $v(X)=v_{\sigma, \alpha}(X)$. Define the function $u$ from $T_{\delta_{2}}^{*}$ to $R$ by the equation

$$
u(X)=\int_{\substack{|Z-X|<\rho_{2} \\ Z \in \sigma}} g_{\alpha}(X-Z) d Z .
$$

Note that $u(X)$ differs from $u_{\sigma, \alpha}(X)$ on $T_{\delta_{2}}^{*}$ by a bounded function. At this point, we note that the assertions (6a) and (6b) remain valid if $u(X)$ is replaced by a function which differs from it by a bounded function. Therefore, we may prove Theorem 1 for $u(X)$ in place of $u_{o, \alpha}(X)$. For $X \in T_{\delta_{2}}^{*}, \sqrt{y(X)}<r<\rho_{2}$, define

$$
\bar{g}(r)=\max _{Z \in S_{r, X}}\left|g_{\alpha}(X-Z)\right| \text {. }
$$

For $X \in T_{\delta_{2}}^{*}$, let

$$
\begin{aligned}
u_{1}(X) & =\int_{\substack{\sqrt{y(X)} \\
Z \in \sigma}} \underset{Z-X \mid<\rho_{2}}{ } g_{\alpha}(X-Z) d Z \\
& =\int_{r=\sqrt{y(X)}}^{\rho_{2}} \int_{\zeta \in S_{r, X}}\left[g_{\alpha}(X-\zeta) / \theta(X, \zeta)\right] d \zeta d r .
\end{aligned}
$$

Using the estimate for $\theta(X, Z)$ with $X \in T_{\delta_{1}}$ and the definition of $\bar{g}$, we have

$$
\left|u_{1}(X)\right|<\int_{r=\sqrt{y(X)}}^{\rho_{2}} 4 \omega_{d-1} r^{d-1} \bar{g}(r) d r
$$

Moreover, from the homogeneity of $g(X)$ we have $\bar{g}(r)<C_{n, m} r^{2-n-m}$ with $C_{n, m}>$ 0 . Combining these two estimates, we have

$$
\left|u_{1}(X)\right|<4 C_{n, m} \omega_{d-1}[y(X)]^{(2-n-m+d) / 2}=4 C_{n, m} \omega_{d-1}[y(X)]^{(2-k-m) / 2} .
$$

Therefore, $\lim [y(X)]^{m+k-2} u_{1}(X)=0$ as $X \rightarrow \sigma_{1}$ through $T_{\delta_{2}}$. Thus we may prove 
Theorem 1 for $u_{2}(X)=u(X)-u_{1}(X)$. For $X \in T_{\delta_{2}}$, let $B_{r, X}=\left\{Z\left|Z \in \sigma_{1},\right| X-\right.$ $Z \mid \leqslant r$. We may equally well define $u_{2}(X)$ by $u_{2}(X)=\int_{B_{\sqrt{y(X), X}}} g_{\alpha}(X-Z) d Z$. For $X \in T_{\delta_{2}}^{*}$, define $W(X)=Y(X) / y(X)$. For $Z \in \sigma_{1}$, let $Q_{Z}=P_{Z}-Z$ (that is, translate $P_{Z}$ to the origin) be the tangent space to $\sigma_{1}$ at the point $Z$. Let

$$
D_{\delta}^{*}=\left\{\langle y(X), Z(X), W(X)\rangle \mid X \in T_{\delta}^{*}, 0<\delta<\delta_{2}\right\} .
$$

$D_{\delta}^{*}$ is an $n$-dimensional subset of $R \times \sigma_{1} \times S^{(n-1)}$. Note that $W(X)$ is perpendicular to $Q_{Z(X)}$. Therefore, the vector $W(X)$ is bound by the above relation and thus has $k-1$ degrees of freedom. The transformation $\langle y, Z, W\rangle$ of $X \in T_{\delta_{2}}^{*}$ into $D_{\delta}^{*}$ defined by $y=y(X), Z=Z(X), W=W(X)$ is a diffeomorphism.

Define the functions $u^{*}, v^{*}$ from $D_{\delta_{2}}^{*}$ into $R$ by $u^{*}(y, Z, W)=y^{k+m-2}$. $u_{2}(X(y, Z, W))$ and $v^{*}(y, Z, W)=y^{k+m-2} v(X(y, Z, W))$. By the homogeneity of the kernel $g_{\alpha}$ (order $2-n-m$ ), we have

$$
\begin{aligned}
v^{*}(y, Z, W) & =y^{k+m-2} \int_{P_{\mathrm{Z}(X)}} g_{\alpha}(X-\lambda) d \lambda=y^{-d} \int_{P_{\mathrm{Z}(X)}} g\left(\frac{X-\lambda}{y}\right) d \lambda \\
& =y^{-d} \int_{P_{\mathrm{Z}(X)}} g_{\alpha}\left(\frac{1}{y}[(X-Z)-(\lambda-Z)]\right) d \lambda=\int_{Q_{\mathrm{Z}(X)}} g_{\alpha}[W-\eta] d \eta .
\end{aligned}
$$

Note that $v^{*}$ only depends on $Z, W$. We shall, therefore, write $v^{*}(y, Z, W)$ as $v^{*}(Z, W)$.

For $0<\delta \leqslant \delta_{2}$, let $D_{\delta}=\overline{D_{\delta}^{*}}$. For $X \in T_{\delta_{2}}^{*}$, define

$$
\sigma_{x}=(B \sqrt{y(x)}, x-Z(X)) / y(X)=\{U \mid U=\eta / y, \eta \in B \sqrt{y(x)} x-Z(X)\},
$$

where $B_{r, X}-Z(X)$ is the translation of $B_{r, X}$ by $-Z(X)$. Let $\left\{y_{i}, Z_{i}, W_{i}\right\}_{i=1}^{\infty}$ be a sequence with the property

$$
\lim _{i \rightarrow \infty} y_{i}=0, \lim _{i \rightarrow \infty} Z_{i}=Z_{0}, \quad \lim _{i \rightarrow \infty} W_{i}=W_{0} .
$$

We shall prove that $\lim _{i \rightarrow \infty} u^{*}\left(y_{i}, Z_{i}, W_{i}\right)=v^{*}\left(Z_{0}, W_{0}\right)$.

$$
\begin{aligned}
u^{*}(y, Z, W) & =y^{k+m-2} \int_{B \sqrt{y(X), x}} g_{\alpha}(X-\lambda) d \lambda=y^{-d} \int_{B \sqrt{y(x)}, x} g_{\alpha}\left(\frac{X-\lambda}{y}\right) d \lambda \\
& =y^{-d} \int_{B \sqrt{y(X)}, x} g_{\alpha}\left(\frac{(X-Z)-(\lambda-Z)}{y}\right) d \lambda=\int_{\sigma_{X}} g_{\alpha}[W-\eta] d \eta .
\end{aligned}
$$

By definition, the surface $\sigma_{X\left(y_{i}, Z_{i}, W_{i}\right)}$ approaches $Q_{z_{0}}$ in $C^{\infty}$ as $i \rightarrow \infty$. Furthermore, $W_{0}$ is normal to $Q_{z_{0}}$ and $W_{0}$ is of unit length, so that

$$
\left|g_{\alpha}\left(W_{0}-\eta\right)\right|<2\left(1+\left|Z_{0}-\eta\right|^{2}\right)^{(2-n-m) / 2}
$$

which is an integrable function over $Q_{z_{0}}$. Therefore, by the dominated convergence theorem, $\lim _{i \rightarrow \infty} u^{*}\left(y_{i}, Z_{i}, W_{i}\right)=v^{*}\left(Z_{0}, W_{0}\right)$. We may now extend $u^{*}$ to $D_{\delta_{2}}$ as a continuous function. Since $D_{\delta_{2}}$ is compact, $u^{*}$ is uniformly continuous on $D_{\delta_{2}}$. This proves the theorem for $m+k>3$.

CASE $2(k=2, m=0)$. Fix a positive $\delta_{3}, \delta_{3}<\delta_{2}$, so that for $X \in T_{\delta_{3}}^{*}$ we have $\left|\operatorname{grad} u_{\sigma, \alpha}(X)+\omega_{d-1} W / y\right|<\varepsilon / 2 y(X)$. 
Define $\sigma^{*}=\left\{X \mid X \in R^{n}, \operatorname{dist}(X, \sigma)=\delta_{3}\right\}$ and $b=\max _{X \in \sigma^{*}}\left|u_{\sigma, \alpha}(X)+\omega_{d-1} \ln \delta_{3}\right|$. Clearly $b$ exists since $\sigma^{*}$ is compact. Let $\delta_{4}=\min \left(\delta_{3}, \exp (-2 b / \varepsilon)\right)$. Define the function $A$ from $T_{\delta_{3}}^{*}$ to $\sigma^{*}$ by $A(X)=Z(X)+\delta_{3} W(X)$. For $X \in T_{\delta_{4}}^{*}$

$$
\begin{aligned}
\left|u_{\sigma, \alpha}(X)+\omega_{d-1} \ln y(X)\right| \leqslant & \left|u_{\sigma, \alpha}(A(X))+\omega_{d-1} \ln \delta_{3}\right| \\
& +\left|\int_{A(X)}^{X}\left(\operatorname{grad} u_{\sigma, \alpha}(\eta)+\omega_{d-1} \frac{W(\eta)}{y(\eta)}\right) \cdot d \eta\right| \\
\leqslant & b+\int_{y(X)}^{\delta_{3}} \frac{\varepsilon}{2 t} d t<b-\frac{\varepsilon}{2} \ln y(X)<-\varepsilon \ln y(X) .
\end{aligned}
$$

This completes the proof of Theorem 1 .

2.1. Elementary condition numbers. It is difficult to provide a direct construction of a harmonic realization of every set of condition numbers. We shall first realize condition numbers which satisfy stronger conditions than the inequalities of (1). In addition, we shall always assume, with no loss of generality, that the domains in question are connected, i.e., $R_{0}=1$. We term nonnegative integers $1, R_{1}, R_{2}, \mu_{0}, \mu_{1}$, $\mu_{2}, M_{1}, M_{2}$, elementary condition numbers if they satisfy the following equation.

$$
\mu_{0}=1, \quad R_{2}=0, \quad \mu_{1}<R_{1} .
$$

THEOREM 2. Suppose $1, R_{1}, R_{2}, \mu_{0}, \mu_{1}, \mu_{2}, M_{1}, M_{2}$ is a set of elementary condition numbers. Then there exists a function, $u$, and a domain, $\Omega$, which comprise a harmonic realization of the numbers $1, R_{1}, R_{2}, \mu_{0}, \mu_{1}, \mu_{2}, M_{1}, M_{2}$.

The proof of Theorem 2 is entirely constructive. The function, $u$, will be essentially the electrostatic potential of a charged arc, $\gamma$. The geometric form of $\gamma$ will depend upon the integer $M_{1}$.

We shall construct $\Omega$ by determining its boundary. The tools used to do this are essentially analytic, but underlying the analysis are choices which depend on the elementary condition numbers which are to be realized.

2.2. Standard approximation. Lemma 1 and its corollaries assert that the character of a n.d. critical point remains unchanged under small perturbations.

LEMMA 1. Suppose $\Omega$ is a bounded open subset of $R^{n}$ and $u$ is a real-valued function in the class $C^{2}(\bar{\Omega})$. Moreover, assume that $u$ has exactly one critical point, $P$, in $\bar{\Omega} ; P$ is contained in $\Omega$ and is $n . d$. of index $k$. If $u_{1}, u_{2}, \ldots$ is a sequence of real-valued functions in $C^{2}(\bar{\Omega})$ which converges to $u$ in $C^{2}$ uniformly on $\bar{\Omega}$, then there exists a number, $N$, such that for each $i>N, u_{i}$ has exactly one critical point, $P_{i} ; P_{i}$ is n.d. of index $k$ and $\lim _{i \rightarrow \infty} P_{i}=P$.

Proof. First use the inverse function theorem to solve the equation $\operatorname{grad}\left(u_{i}\left(P_{i}\right)\right)$ $=0$ near $P$. This is always possible for $i$ sufficiently large because $P$ is a n.d. critical point of $u$ and the convergence of the functions $u_{i}$ is in $C^{2}$. Moreover, this solution, $P_{i}$, is unique within some fixed neighborhood $V$ of $P$. Since $\bar{\Omega}-\dot{V}$ is compact and convergence of $u_{i}$ is uniform in $C^{2}$, for $i$ is sufficiently large the functions, $u_{i}$, cannot have critical points in $\bar{\Omega}-\stackrel{\circ}{ }$. This completes the proof. 
COROllary 1. Suppose $\Sigma$ is a compact n-manifold, perhaps with a boundary. Let $u$ be a n.d. function on $\Sigma$ with critical points $P_{1}, \ldots, P_{M} ; P_{j}$ does not belong to the boundary of $\Sigma, j=1, \ldots, M$. Suppose $k_{j}=$ index of $P_{j}$ for $j=1, \ldots, M$. If $u_{1}$, $u_{2}, \ldots$ is a sequence of functions with $u_{i}$ in class $C^{2}$ on $\Sigma$ which converges uniformly to $u$ in $C^{2}$, then there exists a number, $N$, such that $i>N$ implies $u_{i}$ has exactly $M$ critical points, $P_{j}^{i} ; P_{j}^{i}$ is n.d. with index $k_{j}$ and $\lim _{i \rightarrow \infty} P_{j}^{i}=P_{j}, j=1, \ldots, M$. This corollary follows by applying Lemma 1 in local coordinates neighboring each critical point $P_{j}$.

Corollary 2. Suppose $\Sigma$ is as prescribed in Corollary 1. Let $\psi$ be an imbedding of $\Sigma$ into $R^{q} ; \psi$ is of class $C^{2}$. Let $\Sigma^{\prime}=\psi(\Sigma)$. Suppose $u$ is a function of class $C^{2}$ defined in a $q$-dimensional neighborhood of $\Sigma^{\prime}$. Suppose the restriction of $u$ to $\Sigma^{\prime}$ is n.d. with critical points, $P_{1}, \ldots, P_{M}$ with index of $P_{j}=k_{j}$. Moreover, suppose $\psi^{-1}\left(P_{j}\right)$ does not belong to the boundary of $\Sigma, j=1, \ldots, M$. Let $\psi_{1}, \psi_{2}, \ldots$ be a sequence of $C^{2}$ imbeddings of $\Sigma$ into $R^{q}$ which converges to $\psi$ uniformly in $C^{2}$. Let $\Sigma_{i}=\psi_{i}(\Sigma)$. Then there exists a number $N$ such that for $i>N$ the restriction of $u$ to $\Sigma_{i}$ has exactly $M$ critical points, $P_{j}^{i}$. Each $P_{j}^{i}$ is n.d. with index $k_{j}$, and the $\lim _{i \rightarrow \infty} P_{j}^{i}=P_{j}, j=$ $1, \ldots, M$.

Define a $C^{k}$ family of functions $\mathscr{F}$ to be any collection of functions indexed by a real parameter such that each function $f_{e}$ is of class $C^{k}$ and each derivative of $f_{e}$ of order $k$ or less is a jointly continuous function of the $n+1$ variables, $x_{1}$, $x_{2}, \ldots, x_{n}, \varepsilon$.

Corollary 3. Suppose $\mathcal{Q}$ is a $C^{2}$ family of functions indexed by $\varepsilon, 0<\varepsilon<1$. Assume each $u_{\varepsilon}$ is defined in a neighborhood of the origin in $R^{n}$ and that the origin is an ordinary point of $u_{0}$. Let $S$ be any compact submanifold of $R^{n}$ of class $C^{2}$. Define $L$ a real-valued linear function on $R^{n}$ by $L(X)=\left(\left(\operatorname{grad} u_{0}\right)(0)\right) \cdot X$. Let $S_{e}=$ the set $\varepsilon S$ for $\varepsilon$ a positive number. If $L$ is $n$.d. on $S$ with type numbers $J_{0}, \ldots, J_{k}$, then there exists a positive number $\varepsilon_{0}$ such that for each $\varepsilon, 0<\varepsilon<\varepsilon_{0}$, the restriction of $u_{e}$ to $S_{e}$ is $n . d$. and has type numbers $J_{0}, \ldots, J_{k}$.

Proof. Define the $C^{2}$ family of functions $\mathscr{F}$ with $f_{\varepsilon}$ defined in a neighborhood of the origin in $R^{n}$ for positive $\varepsilon$ by $f_{\varepsilon}(X)=(1 / \varepsilon)\left(u_{\varepsilon}(\varepsilon X)-u_{\varepsilon}(0)\right)$. It is clear that $f_{\varepsilon}$ tends to $L$ in $C^{2}$ uniformly on compact subsets of $R^{n}$ as $\varepsilon \rightarrow 0^{+}$. In particular, $f_{\varepsilon} \rightarrow L$ in $C^{2}(S)$ uniformly. Therefore, by Corollary 1 of Lemma 1 , there exists an $\varepsilon_{0}$ such that for all positive $\varepsilon, \varepsilon<\varepsilon_{0}$, the type numbers of $f_{\varepsilon}$ on $S$ are identical to those of $L$ on $S$. On the other hand, for any fixed $\varepsilon$, the function $f_{\varepsilon}$ differs from $u_{e}$ by a dilation, subtraction of a constant and multiplication by another constant. Therefore, the type numbers of $u_{\varepsilon}$ on the set $\varepsilon S$ must be identical to those of $f_{\varepsilon}$ on $S$. This proves the corollary.

2.3. Construction of $u$ for $M_{1} \neq 0$. A harmonic function $u$, defined on an open subset of $R^{3}$, will be found which has $2 M_{1}$ critical points, half having index 1 and the remainder having index 2. An arc is a one-dimensional $C^{\infty}$ submanifold of $R^{3}$ with boundary, which is the homeomorphic image of a closed interval. We often shall use the same symbol to denote the arc, the point set and its parametric 
representation. We parametrize an arc $\gamma$ by $\gamma(t)=\left(\gamma_{1}(t), \gamma_{2}(t), \gamma_{3}(t)\right), t \in[-a, a]$. Fix the arc $\gamma$ and let $u^{\gamma}(X)$ be the function defined by

$$
u^{\gamma}(X)=-\int_{\gamma} g(X-Z) d Z, \quad X \in R^{3}-\{\gamma\}
$$

We require that the arc $\gamma$ satisfy the following conditions.

(a) $\gamma_{3}=0$ and $\gamma(0)=0$

(b) $\gamma_{1}$ is even and $\gamma_{2}$ is odd

(c) $\gamma_{1}^{\prime}(t)>0$ for $0<t<a$

(d) there are real numbers $q_{i}$, $q_{i}^{*}$ with $0<q_{i}<q_{i}^{*}<q_{i+1}<\gamma_{1}(a)$,

$i=1,2, \ldots, M_{1}$, such that $u^{\gamma}\left(q_{i}, 0,0\right)<-2$ and $u^{\gamma}\left(q_{i}^{*}, 0,0\right)>-1$.

It is clear that an arc $\gamma$ may be found such that the conditions (a), (b), and (c) of (12) are satisfied. Theorem 1 implies that $u^{\gamma} \rightarrow-\infty$ as $X \rightarrow Z, Z \in \dot{\gamma}$ where $\dot{\gamma}$ is the interior of $\gamma$. Therefore, condition (d) of (12) may be satisfied by an arc like the one shown in Figure 2. Note that $\gamma_{2} \neq 0$ for $t \neq 0$. It should be pointed out that the conditions (a) and (b) are designed to avoid unnecessary complications, whereas (c) and (d) are essential. Violation of (c) introduces critical points that are extraneous. The function $u^{\gamma}$ has the following symmetry property.

$$
u^{\gamma}\left(x_{1}, x_{2}, x_{3}\right)=u^{\gamma}\left(x_{1},-x_{2}, x_{3}\right)=u^{\gamma}\left(x_{1}, x_{2},-x_{3}\right) \text {. }
$$

By (12c), a function $f_{\gamma}$ can be defined on $\left[0, \gamma_{1}(a)\right]$ by

$$
f_{\gamma}\left(\gamma_{1}(t)\right)=\gamma_{2}(t), \quad t \in[0, a] \text {. }
$$

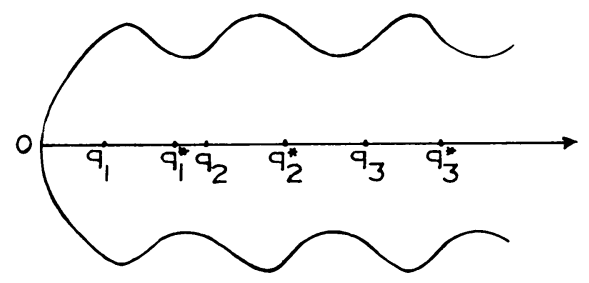

FIGURE 2

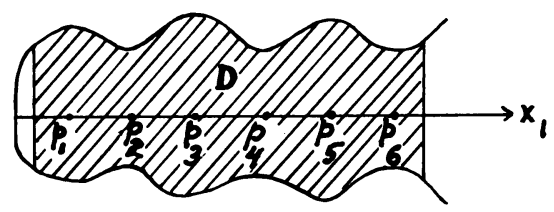

Figure 3

By Theorem 1, it is possible to choose a positive number $\delta_{1}<q_{1}$ such that for $X=\left(x_{1}, x_{2}, x_{3}\right), 0<x_{1}<\delta_{1},\left|x_{2}\right|<f_{\gamma}\left(x_{1}\right)$ and $x_{3}=0$,

$$
\left|\operatorname{grad}\left(u^{\gamma}(X)\right)\right|>1 \text {. }
$$


Let $a_{1}$ be a number such that $q_{M_{1}+1}<a_{1}<\gamma_{1}(a)$. Let $D$ and $D^{*}$ be the sets defined by

$$
\begin{aligned}
& \text { (a) } D=\left\{X\left|X \in R^{3}, \delta_{1}<x_{1}<a_{1},\right| x_{2} \mid<f_{\gamma}\left(x_{1}\right), x_{3}=0\right\} \text {, } \\
& \text { (b) } D^{*}=\left\{X\left|X \in R^{3}, 0<x_{1}<a_{1},\right| x_{2} \mid<f_{\gamma}\left(x_{1}\right)\right\}
\end{aligned}
$$

and $h$ be the restriction of $u^{\gamma}$ to the set $\left\{X \mid X \in R^{3}, \delta_{1}<x_{1}<a_{1}, x_{2}=0=x_{3}\right\}$. By Lemma 6.2 of [3], it is possible to choose a real number $\beta$ such that

(16)

$$
\begin{aligned}
& \text { (a) }|\beta|<\frac{1}{2}\left(1 /\left(1+a_{1}\right)\right) \text { and } \\
& \text { (b) } h+\beta x_{1} \text { is n.d. }
\end{aligned}
$$

Let $p_{1}<p_{2}<\cdots<p_{K}$ be the critical points of the function $h+\beta x_{1}$. By (12d) and (16a), $K>2 M_{1}$. Decrease $a_{1}$, if necessary, so that the function $h+\beta x_{1}$ has exactly $2 M_{1}$ critical points in $\left(\delta_{1}, a_{1}\right)$ and $a_{1}$ is not a critical point. By Theorem $1, p_{1}$ is a maximum of $h+\beta x_{1}$. Therefore

$$
p_{i} \text { is a maximum if } i \text { is odd and a minimum if } i \text { is even. }
$$

Let

$$
P_{i}=\left(p_{i}, 0,0\right)
$$

and

$$
b=\sup _{X \in D}\left\{\left(\partial^{2} u^{\gamma} / \partial x_{2}^{2}\right)(X)\right\} .
$$

Then $b$ exists since (5) and (12c) imply that

$$
\left(\partial^{2} u^{\gamma} / \partial x_{2}^{2}\right)(X) \rightarrow-\infty \quad \text { as } X \rightarrow \gamma \text { through } D \text {. }
$$

Define the function $\psi$ by $\psi(X)=(1+|b|)\left(x_{3}^{2}-x_{2}^{2}\right) / 2$ and let

$$
u(X)=u^{\gamma}(X)+\beta x_{1}+\psi(X) \text {. }
$$

The function $u(X)$ has the following properties.

(22) (a) $u$ is defined and harmonic on $D^{*}$.

(b) The asymptotic representation which Theorem 1 asserts for $u^{\gamma}$ and its derivatives is valid for $u$.

(c) $u$ has the symmetry properties of $u^{\gamma}$.

(d) (i) $\left(\partial^{2} u / \partial x_{2}^{2}\right)(X)<-1, X \in D$,

(ii) $\left(\partial^{2} u / \partial x_{3}^{2}\right)(X)>a_{2}>0, X \in D^{*}$ and $x_{3}=0$.

(e) (i) $\operatorname{sgn}\left(\partial u / \partial x_{2}\right)(X)=-\operatorname{sgn}\left(x_{2}\right), X \in D$,

(ii) $\operatorname{sgn}\left(\partial u / \partial x_{3}\right)(X)=\operatorname{sgn}\left(x_{3}\right), X \in D^{*}$.

(f) The only critical points of $u$ in $D^{*}$ are the $p_{i}$ and each $p_{i}$ is n.d. with index 2 or 1 according as $i$ is odd or even, respectively.

It should be noted that (c) and (d) can be used to verify (e). Moreover, (e) implies (f).

2.4. Construction of $\Omega$ for $M_{1} \neq 0$. We adopt the notation of the preceding section with the following exception. The symbols $P_{i}, p_{i}$ are no longer defined for $i>2 M_{1}$. 
In this section a simple plane closed curve, $\nu$, of the topological type of the circle will be constructed. The curve $\nu$ will lie in $W=\left\{X \mid X \in D^{*}, x_{3}=0\right\}$. In addition, the curve $\nu$ will satisfy the following. (i) $\nu$ is of class $C^{\infty}$, (ii) $\nu$ surrounds the points $P_{i}, i=1,2, \ldots, 2 M_{1}$, (iii) the function $u$ restricted to $\nu$ has exactly two critical points, $P_{0}$ and $P_{2 M_{1}+1}$, (iv) of these two points, only $P_{0}$ is entrant in the appropriate sense. The asymptotic estimates of $u$ near the curve $\gamma$, obtained in Theorem 1 , will be used to accomplish this construction. The nontrivial part of the curve $\nu$ will, in fact, be the solution of a certain initial value problem which, for notational convenience, is set in the complex plane. Therefore, let $W$ be identified with a region in the complex plane with $\xi=x_{1}+i x_{2}$.

Define the function $\theta_{1}$, mapping $W-\cup_{j=1}^{2 M_{1}} p_{j}$ into the unit circle $S^{1}$, as follows.

$$
\theta_{1}(\xi)=\operatorname{grad}(u(\xi)) /|\operatorname{grad}(u(\xi))|
$$

where $\operatorname{grad}(u)$ is taken to mean $\partial u / \partial x_{1}+i\left(\partial u / \partial x_{2}\right)$. Define the function $\theta\left(\varepsilon, t, t_{0}, \xi\right)$ mapping $R^{2} \times\{\xi|\xi \in W,| \operatorname{grad}(u(\xi)) \mid>1\}$ into $S^{1}$ by the following:

$$
\theta\left(\varepsilon, t, t_{0}, \xi\right)=e^{-i \sin ^{-1}\left[2 t /\left(\left(1+t^{2}\right)^{2}|\operatorname{grad}(u(\xi))|\right)\right]} \text { for }|t| \leqslant t_{0}
$$

For the time being it will be assumed that $t_{0}$ is infinite and therefore the function $\theta\left(\varepsilon, t, t_{0}, \xi\right)$ will be independent of $t_{0}$ and $\varepsilon$. Define the function $\nu_{\delta}$ as follows.

$$
(d / d t)\left(\nu_{\delta}\right)=i \theta_{1}\left(\nu_{\delta}\right) \theta\left(\varepsilon, t, \infty, \nu_{\delta}\right), \quad \nu_{\delta}(0)=\delta .
$$

Obviously the path $\nu_{\delta}$ is parameterized by arc length. It is a straightforward matter to compute the following.

$$
\begin{aligned}
(d / d t) u\left(\nu_{\delta}(t)\right) & =\operatorname{Re}\left(\operatorname{grad}(u) \overline{(d / d t) \nu_{\delta}}\right)=-\operatorname{Re}\left(i \theta_{1} \bar{\theta}_{1} \overline{\theta(\varepsilon)}\right) \cdot|\operatorname{grad}(u(\xi))| \\
& =2 t /\left(1+t^{2}\right)^{2}
\end{aligned}
$$

Therefore

$$
u\left(\nu_{\delta}(t)\right)=u(\delta)+\int_{0}^{t} \frac{2 s d s}{\left(1+s^{2}\right)^{2}}=u(\delta)+1-\frac{1}{1+t^{2}} .
$$

It is useful to observe that $\nu_{\delta}$ is a level curve of a function which differs from $u$ by a bounded quantity. By Theorem $1, u(X) \rightarrow-\infty$ and $\operatorname{grad}(u) \rightarrow 2 Y / y^{2}$ as $X$ approaches $\gamma$. Therefore, the tangent vectors to $\nu_{\delta}$, at $X$, and $\gamma$, at $Z(X)$, are essentially parallel. Let $T^{*}=\inf \left\{t>0 \mid \nu_{\delta}(t) \notin W\right\}$. It is clear from the preceding remarks that, for $\delta$ sufficiently small, $T^{*}$ exists and that $\nu_{\delta}$ approaches $\gamma$ in $C^{1}$ uniformly for $0 \leqslant t \leqslant T^{*}$ as $\delta \rightarrow 0^{+}$. Therefore, it is possible to find small positive numbers $\delta_{1}, \delta_{2}$ such that the following conditions are satisfied.

(28) (a) $\operatorname{Re}\left(\nu_{\delta_{2}}(t)\right)$ is $1-1$ for $\delta_{1} \leqslant t \leqslant T^{*}$.

(b) $\operatorname{Im}\left(\nu_{\delta_{2}}(t)\right)$ is positive for $0<t<T^{*}$.

(c) $\delta_{2}<p_{1}$.

(d) $\delta_{1}$ depends on $\delta_{2}$ and, due to $C^{1}$ convergence of $\nu_{\delta}$ to $\gamma, \delta_{1} \rightarrow 0^{+}$as $\delta_{2} \rightarrow 0^{+}$. Now define $t_{0}>0$ by $\operatorname{Re}\left(\nu_{\delta_{2}}\left(t_{0}\right)\right)=p_{2 M_{1}}$. That $t_{0}$ is well defined follows from (28a) and (28d). We now extend the definition of $\theta\left(\varepsilon, t, t_{0}, \xi\right)$ for $t>t_{0}$. For $t_{0}<t<t_{0}$ $+\varepsilon$, let $\theta\left(\varepsilon, t, t_{0}, \xi\right)$ satisfy

(29) (a) $\operatorname{Im}\left[\theta\left(\varepsilon, t, t_{0}, \xi\right) \theta_{1}(\xi)\right] \leqslant 0$ with equality at $t=t_{0}+\varepsilon$, 
(b) $\operatorname{Im} \theta\left(\varepsilon, t, t_{0}, \xi\right)<0$.

Moreover we require that for $t>t_{0}+\varepsilon, \theta\left(\varepsilon, t, t_{0}, \xi\right)=-\bar{\theta}_{1}(\xi)$. By further restricting $\delta_{2}$, if necessary, it is possible to find a function $\theta(\varepsilon)$ which satisfies (24), (29) and is of class $C^{\infty}$, for every $\varepsilon>0$. Let

$$
\varepsilon=\frac{1}{2}\left[\min \left(a_{1}-p_{2 M_{1}}, \operatorname{Im}\left(\nu_{\delta_{2}}\left(t_{0}\right)\right) / 2\right)\right]
$$

and $t_{1}=t_{0}+2 \varepsilon$. Define $\hat{\nu}$ for $0 \leqslant t \leqslant t_{1}$ by

$$
(d / d t) \hat{\nu}(t)=i \cdot \theta_{1}(\hat{\nu}) \cdot \theta\left(\varepsilon, t, t_{0}, \hat{\nu}\right), \quad \nu(0)=\delta_{2} .
$$

Extend $\hat{\nu}$ for $|t| \leqslant t_{1}$ by $\hat{\nu}(-t)=\overline{\hat{\nu}(t)}$. By (29) and (22e(i)), $u(\nu(t))$ increases for $0 \leqslant t \leqslant t_{1}$. Therefore this section of $\hat{\nu}$ is an arc. By symmetry, $\hat{\nu}\left[-t_{1}, 0\right]$ is an arc. By the choice of $\delta_{2}$ and $t_{1}$, these two branches of $\hat{\nu}$ intersect at only one point, $\hat{\nu}(0)=\delta_{2}=p_{0}$. Therefore, $\left\{\hat{\nu}\left(\left[-t_{1}, t_{1}\right]\right)\right\}$ is a continuous arc, and by (25) is in fact of class $C^{\infty}$. Extend $\hat{\nu}$ as shown in Figure 4 to be a $C^{\infty}$ closed curve of the topological type of the circle. Call this curve $\nu$. Let $p_{2 M_{1}+1}=\nu \cap\left\{\xi \subset R \mid \xi>p_{0}\right\}$.

(31) By (29) and (22e(i)), the only critical points of the restriction of $u$ to $\nu$ are $p_{0}$, the minimum, and $p_{2 M_{1}+1}$, the maximum. At both points the gradient of $u$ lies on the positive, real axis.

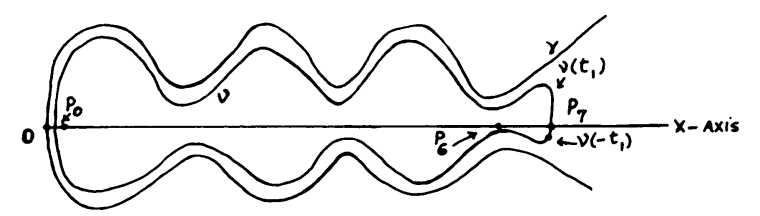

FIgURE 4. Drawn for $M_{1}=3$

In the succeeding discussion the complex notation is dropped, but $X$ is still constrained to lie in the $x_{1}-x_{2}$ plane. Define $W^{\prime}$ to be the closed bounded subset of the plane whose boundary is the closed curve $\nu$. Select $\mu_{1}$ distinct points $X^{i} ; X^{i}$ belongs to $\mathscr{W}^{\prime}-\left\{\cup_{j} P_{j}\right\}, i=1, \ldots, \mu_{1}, j=1, \ldots, 2 M_{1}$. For positive numbers $\delta_{i}$ define $\nu_{\delta_{i}}$ to be circles of radius $\delta_{i}$, centered at the points $X^{i}, i=1, \ldots, \mu_{1}$. Define $\hat{u}=u$ restricted to $W$. For positive numbers $\delta_{j}^{\prime}$, let $\nu_{\delta_{j}^{\prime}}=$ the circle of radius $\delta_{j}^{\prime}$ centered at the point $P_{2 j-1}, j=1, \ldots, R_{1}-\mu_{1}$. Let $\nu^{*}=\left\{\nu \cup_{i} \nu_{\delta_{i}} \cup_{j} \nu_{\delta, j}\right\}, i=$ $1, \ldots, \mu_{1}, j=1, \ldots, R_{1}-\mu_{1}$. Let $W^{*}=$ the closed bounded connected region of the plane whose boundary is $\nu^{*}$. We term critical points of $\hat{u}$ restricted to $\nu^{*}$ to be entrant or emergent with respect to the region $W^{*}$.

Since $X^{i}, i=1,2, \ldots, \mu_{1}$, are not critical points, according to Corollary 3 of Lemma 1 , it is possible to choose the numbers, $\delta_{i}, \delta_{j}^{\prime}$, so small that $\hat{u}$ restricted to $\nu_{\delta_{i}}$ is n.d. and has precisely one entrant critical point, $P_{i}{ }^{1}$, with index of $P_{i}{ }^{1}=1$; $\nu_{\delta_{j}^{\prime}}$ is emergent, $i=1, \ldots, \mu_{1}, j=1, \ldots, R_{1}-\mu_{1}$. It is clear from (31) that the restriction of $\hat{u}$ to $\nu$ is n.d. and has precisely one entrant critical point, namely $\boldsymbol{P}_{0}=$ $\left\langle p_{0}, 0,0\right\rangle$, with index of $P_{0}=0$.

We now return to a 3-dimensional setting, and define $\Sigma=\{X$ belongs to $R^{3} \mid\left\langle x_{1}, x_{2}, 0\right\rangle$ belongs to $\left.\nu^{*}\right\}$. Define $\Omega^{*}=\left\{X\right.$ belongs to $R^{3} \mid\left\langle x_{1}, x_{2}, 0\right\rangle$ belongs to $\left.W^{*}\right\}$. We now term critical points of the restriction of $u$ to $\Sigma$ to be entrant or emergent with respect to the region $\Omega^{*}$. 
It is clear from (22e(i)) that every critical point of the restriction of $u$ to $\Sigma$ lies on the curve $\nu^{*}$, and from (22d(ii)), together with the preceding discussion, that the restriction of $u$ to $\Sigma$ is n.d. Moreover, the entrant critical points of the restriction of $u$ to $\Sigma$ correspond in position and index to the entrant critical points of the restriction of $\hat{u}$ to $\nu^{*}$.

Define $\Sigma_{1}=\left\{X\right.$ belongs to $\left.\Sigma \mid x_{3}^{2} \leqslant 1\right\}$. Let $f_{1}$ be a real-valued function defined on $W^{*}$ subject to the following conditions.

(32) (a) The function $f_{1}$ is continuous on $W^{*}$.

(b) $f_{1} \equiv 1$ on $\nu^{*}$.

(c) For $j=R_{1}-\mu_{1}+1, \ldots, R_{1}-\mu_{1}+\mu_{2}, f_{1}\left(P_{2 j-1}\right)<0$.

(d) For $j=1, \ldots, M_{1}, f_{1}\left(P_{2 j}\right)>0$.

(e) For $j=R_{1}-\mu_{1}+\mu_{2}+1, \ldots, M_{1}, f_{1}\left(P_{2 j-1}\right)>0$.

(f) $\Sigma_{1} \cup$ \{graph of $f_{1}$ \} is a $C^{\infty}$ surface with boundary and it is homeomorphic to $W^{*}$.

Let $g_{1}$ be a real-valued function defined on $W^{*}$ subject to the following conditions.

(33) (a) The function $g_{1}$ is continuous on $W^{*}$.

(b) $g_{1} \equiv-1$ on $\nu^{*}$.

(c) $g_{1}<\min \left(0, f_{1}\right)$.

(d) The graph of $f_{1} \cup$ the graph of $g_{1} \cup \Sigma_{1}$ is a $C^{\infty}$ manifold of dimension 2 of the topological type of the connected sum of $R_{1}$ tori.

Define $f_{\varepsilon}=\varepsilon f_{1}, g_{\varepsilon}=\varepsilon g_{1}$, for $\varepsilon$ any positive number. Let $\tilde{D}$ be a compact subset of $\dot{W}^{*}$ which contains every critical point of $u$ which is contained in $W^{*}$. We may also suppose that $\tilde{D}$ is a 2 -cell with smooth boundary. Define $\psi_{\varepsilon}$, a class $C^{2}$ imbedding of $\tilde{D}$ into $R^{3}$, as follows.

$$
\psi_{\varepsilon}\left(\left\langle x_{1}, x_{2}\right\rangle\right)=\left\langle x_{1}, x_{2}, \varepsilon f_{1}\left(\left\langle x_{1}, x_{2}\right\rangle\right)\right\rangle .
$$

By Lemma 1, Corollary 2, for every positive $\varepsilon<\varepsilon_{0}$, the restriction of $u$ to $\left\{\psi_{e}(\tilde{D})\right\}$ has a finite critical set, $P_{i}^{\varepsilon}, i=2\left(R_{1}-\mu_{1}\right)+1, \ldots, 2 M_{1}$, or $i=2 j, j=1, \ldots, R_{1}$ $-\mu_{1}$. Moreover the index $\left(P_{i}^{\varepsilon}\right)=\operatorname{index}\left(P_{i}\right)$ and $\lim _{\varepsilon \rightarrow 0} P_{i}^{e}=P_{i}$.

We adopt the following notational convention. If $V$ is a vector quantity equal to $\left\langle v_{1}, v_{2}, v_{3}\right\rangle,(V)_{i}=v_{i}, i=1,2,3$. By $(32 \mathrm{a}), \operatorname{sgn}\left(\left(P_{i}^{e}\right)_{3}\right)=\operatorname{sgn}\left(f_{1}\left(\left\langle\left(P_{i}\right)_{1},\left(P_{i}\right)_{2}\right\rangle\right)\right)$. Let

$$
\Omega_{\varepsilon}=\left\{X \in \Omega^{*} \mid \varepsilon g_{1}\left(\left\langle x_{1}, x_{2}\right\rangle\right)<x_{3}<\varepsilon f_{1}\left(\left\langle x_{1}, x_{2}\right\rangle\right)\right\} \text {. }
$$

Let $\Sigma_{\varepsilon}=$ boundary of $\Omega_{\varepsilon}$. Let $\Sigma_{f}^{e}=$ the graph of the function $\varepsilon f_{1}$ over the region $W^{*}$. Let $\Sigma_{g}^{e}=$ the graph of the function $\varepsilon g_{1}$ over the region $W^{*}$ and $\Sigma_{1}^{e}=\{X \in$ $\left.\Sigma_{1}|| x_{3} \mid<\varepsilon\right\}$. By $(22 \mathrm{e}(\mathrm{ii}))$ and the definitions of $\Sigma_{f}^{e}$ and $\Sigma_{g}^{e}$, it follows that for $X \in \Sigma_{f}^{e} \cup \Sigma_{g}^{e}$, if $X$ is a critical point of the restriction of $u$ to $\Sigma_{e}$,

$$
\operatorname{sgn}(\operatorname{grad}(u) \cdot N(X))=\operatorname{sgn}\left(x_{3}\right) .
$$

It then follows that only the points $P_{2 j-1}^{e}, j=R_{1}-\mu_{1}+1, \ldots, R_{1}-\mu_{1}+\mu_{2}, P_{0}$ and $P_{i}^{\prime}, i=1, \ldots, \mu_{1}$, are entrant critical points of the restriction of $u$ to $\Sigma_{e}$. Moreover, the points, $P_{2 j-1}^{e}, j=R_{1}-\mu_{1}+1, \ldots, R_{1}-\mu_{1}+\mu_{2}$, each have index $=2$. It then follows that $u$ and $\Omega_{\varepsilon}$ comprise a harmonic realization of the elementary condition numbers, $1, R_{1}, R_{2}, \mu_{0}, \mu_{1}, \mu_{2}, M_{1}, M_{2}$. 
2.5. Harmonic realization for $M_{1}=0$. Suppose $M_{1}=0$; then (1) and (11) give

$$
\mu_{2}=M_{2}=R_{1}-\mu_{1}=0 \text {. }
$$

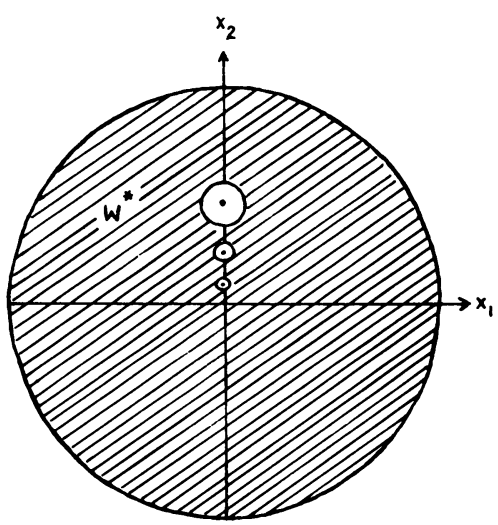

FIGURE 5

Let $u$ be defined by $u(X)=x_{2}$. Let $W^{*}=$ the unit disc in the $x_{1}-x_{2}$ plane $\left\{\cup_{i=1}^{R_{1}}\left\{X\right.\right.$ belongs to $\left.\left.R^{2} \mid x_{1}^{2}+\left(x_{2}-(1 / i+1)\right)^{2}<(i+1)^{-6}\right\}\right\}$. Define a function $f$ on $W^{*}$, subject to the following conditions.

(35) (a) $f$ is continuous on $W^{*}$.

(b) $f$ (boundary of $\left.W^{*}\right) \equiv 0$.

(c) $f\left(\dot{W}^{*}\right)>0$.

(d) graph of $f \cup$ graph of $-f$ is a $C^{\infty}$ manifold homeomorphic to the connected sum of $R_{1}$ tori.

Let $\Omega$ be $\left\{X \in R^{3} \mid\left\langle x_{1}, x_{2}\right\rangle\right.$ belongs to $\dot{W}^{*}$ and $\left.\left|x_{3}\right|<f\left(\left\langle x_{1}, x_{2}\right\rangle\right)\right\}$. The pair $u, \Omega$ provides a harmonic realization of all sets of elementary condition numbers where $M_{1}=0$.

The function $u$ and domain $\Omega$ constructed in $\$ \$ 2.3$ and 2.4 realize any set of elementary condition numbers where $M_{1}$ is greater than 0 . The special case of providing the harmonic realization of elementary condition numbers with $M_{1}$ equal to 0 has just been given in $\$ 2.5$. This completes the proof of Theorem 2.

3.0. In this section techniques will be developed which make it possible to modify the limited examples constructed in \$2.1. These techniques will suffice to show that any set of condition numbers may be realized by an appropriate modification of the harmonic realization of a certain set of elementary condition numbers. Moreover, the necessary modifications are local in nature in that they consist of local changes to the boundary of $\Omega$ or to the function $u$.

\section{1.}

THEOREM 3. Let $\Omega$ be a bounded open subset of $R^{3}$ which contains the origin. Let $\mathscr{F}$ be $a C^{2}$ family of functions defined for $|\varepsilon|<1$ and $X$ contained in a neighborhood of $\bar{\Omega}$. Define the $C^{2}$ family of functions $\mathcal{Q}$ by $u_{\varepsilon}(X)=\varepsilon /|X|$ for $\varepsilon>0$ and $X \in R^{3}$, $|X| \neq 0$. Suppose that $\left|\operatorname{grad}\left(f_{0}\right)\right|>0$ for all $X$ in $\bar{\Omega}$ where $f_{0}$ is the member of $\mathcal{F}$ 
corresponding to $\varepsilon=0$. Define the $C^{2}$ family of functions $\mathcal{V}$ by $v_{e}(X)=f_{e}(X)+$ $u_{\varepsilon}(X)$, for $X$ and $\varepsilon$, simultaneously satisfying the conditions imposed in the definitions of $\mathcal{U}$ and $\mathcal{F}$. Then there exists a positive number $\varepsilon_{0}$ such that for every $\varepsilon, 0<\varepsilon<\varepsilon_{0}$, the following conditions hold.

1. The function $v_{\varepsilon}$ has exactly one critical point $P_{\varepsilon}$ in $\bar{\Omega}$ minus the origin.

2. The point $P_{\varepsilon}$ is a nondegenerate critical point of $v_{\varepsilon}$ with index $\left(P_{e}\right)=2$.

3. $\lim _{\varepsilon \rightarrow 0^{+}} P_{\varepsilon} /(\varepsilon)^{1 / 2}=\operatorname{grad}\left(f_{0}(0)\right) /\left|\operatorname{grad}\left(f_{0}(0)\right)\right|^{3 / 2}$.

Proof. In the following paragraph definitions will be made for the subscript $i=0$, 1. Define $s_{i}^{e}=\left\{X \in R^{3}|| X \mid=\varepsilon^{(3-i) / 5}\right\}$. Let $A^{\varepsilon}$ be the closed bounded subset of $R^{3}$ with boundary $\cup_{i} s_{i}^{\varepsilon}$. Points of $s_{i}^{e}$ shall be termed entrant or emergent with respect to the region $A^{e}$.

By the hypotheses, it is possible to find positive numbers $a$ and $\Delta$ such that for $|\varepsilon|<\Delta$ and $X \in \bar{\Omega},\left|\operatorname{grad}\left(f_{\varepsilon}\right)\right|>a$. On the other hand,

$$
\operatorname{grad}\left(u_{e}\right)=-\varepsilon X /|X|^{3} \text {. }
$$

It follows therefore that for $|X|>\varepsilon^{2 / 5}$,

$$
\left|\operatorname{grad}\left(u_{\varepsilon}\right)\right|<\varepsilon^{1 / 5} \text {. }
$$

Define the positive number $\Delta_{1}$ according to

$$
\Delta_{1}=\min \left\{\Delta,(a / 2)^{5}\right\} \text {. }
$$

Combining the preceding three equations, it follows that if $|X|>\varepsilon^{2 / 5}$ and $0<\varepsilon<$ $\Delta_{1}$, then

$$
\left|\operatorname{grad}\left(v_{e}\right)\right|>a / 2
$$

Define the constant $M$ as follows:

$$
M=\sup \left\{\mid \operatorname{grad}\left(f_{\varepsilon}\right) \| X \in \bar{\Omega} \text { and }|\varepsilon|<\Delta_{1}\right\} .
$$

On the other hand, by (36), it follows that if $0<|X|<\varepsilon^{3 / 5}$ then

$$
\left|\operatorname{grad}\left(u_{e}\right)\right|>\varepsilon^{-1 / 5}
$$

Choose $\Delta_{2}$ as follows:

$$
\Delta_{2}=\min \left\{\Delta_{1},(2 M)^{-5}\right\} .
$$

It follows then from (40), (41), (42), that if $0<\varepsilon<\Delta_{2}$ and $|X|<\varepsilon^{3 / 5}$, then

$$
\left|\operatorname{grad}\left(v_{\varepsilon}\right)\right|>M \text {. }
$$

(39) and (43) imply that for $0<\varepsilon<\Delta_{2}$, the critical points of the function $v_{\varepsilon}$ lie exclusively in the set $A^{\varepsilon}$.

In the following paragraph, the index $i$ may take on the values 0 and 1 . By the definition of the family $\mathcal{Q}$, for every fixed $\varepsilon>0$, the function $u_{\varepsilon}$ is constant on any sphere centered at the origin, and in particular on the spheres $s_{i}^{e}$. Therefore $v_{\varepsilon} \mid s_{i}^{e}$ will possess exactly the same set of type numbers as will $f_{e} \mid s_{i}^{e}$. It follows from Corollary 3 of Lemma 1 that for every sufficiently small positive $\varepsilon$, the type numbers of $f_{\varepsilon} \mid s_{i}^{e}=\left\{\mu_{j}^{e} \mid j=0,1,2\right\}$ are given by

$$
\mu_{0}^{\varepsilon}=\mu_{2}^{\varepsilon}=1, \quad \mu_{1}^{e}=0 .
$$


In the succeeding paragraph, let the index $j$ assume the values $0,1,2$. Define the integers $\nu_{j}^{\varepsilon}$ to be the boundary type numbers of $v_{\varepsilon}$ on $s_{0}^{e}$ and $\gamma_{j}^{e}$ to be the boundary type numbers of $v_{\varepsilon}$ on $s_{1}^{e}$. It follows from (43) that for $0<\varepsilon<\Delta_{2}$, the function $v_{\varepsilon}$ is emergent on all of the boundary $s_{0}^{\varepsilon}$; therefore, $\nu_{j}^{e}=0$. On the other hand, (37) guarantees that for each sufficiently small positive $\varepsilon$, any emergent or entrant critical point of $f_{\varepsilon} \mid s_{1}^{\varepsilon}$ remains respectively an entrant or emergent critical point of $v_{e} \mid s_{1}^{e}$. From this and Corollary 3 of Lemma 1 , it follows that $\gamma_{1}^{e}=\gamma_{2}^{e}=0, \gamma_{0}^{e}=1$. On the other hand, since $\nu_{j}^{e}=0$, the boundary type numbers of $v_{e}$ on $A^{e}$ are $\left\{\gamma_{j}^{\varepsilon} \mid j=0,1,2,\right\}$.

The region $A^{\varepsilon}$ is homotopy equivalent to a 2 -sphere. Therefore if $m_{j}^{\varepsilon}$ are the interior type numbers of $v_{\varepsilon}$ on $A^{\varepsilon}$, then for all sufficiently small positive $\varepsilon$,

$$
\sum_{j=0}^{3}(-1)^{j} m_{j}^{e}=1 .
$$

It will now be shown that for sufficiently small positive $\varepsilon$, the only interior type number of $v_{\varepsilon}$ which can be positive is $m_{2}^{e}$. First compute $H v_{\varepsilon}=H\left(f_{e}+u_{\varepsilon}\right)=$ $\varepsilon W(X) /|X|^{3}+H f_{\varepsilon}=\left(\varepsilon /|X|^{3}\right)\left(W(X)+|X|^{3} H f_{\varepsilon} / \varepsilon\right)$ where $W(X)$ is the $3 \times 3$ matrix $\left(w_{i, j}(X)\right), i, j=1,2,3$ with entries $w_{i, j}(X)=3 x_{i} x_{j} /|X|^{2}-\delta_{i j}$. Clearly index $\left(H v_{\varepsilon}\right)=$ index $\left(W(X)+|X|^{3} H f_{\varepsilon} / \varepsilon\right)$. By the definition of the region $A^{e}$, if $X$ belongs to $A^{\varepsilon}$ then $|X|^{3} / \varepsilon<\varepsilon^{1 / 5}$. A simple computation shows the eigenvalues of the matrix $W(X)$ are $-1,-1,+2$, independent of $X$. Since $\mathscr{F}$ is a $C^{2}$ family of functions, it is clear that $H f_{\varepsilon}$ is bounded in norm. It must then be true that for all sufficiently small positive $\varepsilon$, index $\left(H v_{\varepsilon}\right)=\operatorname{index}(W(X))=2$, for all $X \in A^{e}$. This combined with (45) proves parts 1 and 2 of the theorem. Part 3 follows from an easy calculation.

TheOREM 4. Suppose $\Omega$ is a bounded open subset of $R^{3}$ which contains the origin. Let $\Omega^{\prime}=\Omega$ minus positive $x_{3}$-axis and $d=\left\{X \in \Omega \mid x_{3}=0\right\}$. Let $f$ be a function of class $C^{2}$ on a neighborhood of $\bar{\Omega}$. Define the $C^{2}$ family of functions $\mathcal{Q}$ on $\Omega^{\prime}$ by $u_{\varepsilon}(X)=\varepsilon /\left(x_{1}^{2}+x_{2}^{2}+\left(x_{3}-\varepsilon\right)^{2}\right)^{1 / 2}$ for $\varepsilon>0$. Suppose the restriction of $f$ to $\bar{d}$ has no critical points, and $\partial f / \partial x_{3}<0$ at the origin. Define the family $\mathcal{V}=\mathcal{U}+f$. Then there exists a positive number, $\varepsilon_{0}$, such that for $0<\varepsilon<\varepsilon_{0}$, the function $v_{\varepsilon}$ restricted to $\bar{d}$ has exactly two critical points, $P_{0}^{\varepsilon}, P_{1}^{\varepsilon}$, and the following relations are satisfied for $j=0,1$.

1. $P_{j}^{e}$ is a nondegenerate critical point with index $\left(P_{j}^{e}\right)=2-j$.

2. $\left(\partial v_{\varepsilon} / \partial x_{3}\right)\left(P_{1}^{\varepsilon}\right)<0$ and $\left(\partial v_{\varepsilon} / \partial x_{3}\right)\left(P_{0}^{\varepsilon}\right)>0$.

3. $\lim _{\varepsilon \rightarrow 0} P_{j}^{\varepsilon} / \varepsilon^{1 / 2}=j((\operatorname{grad} f \mid \bar{d})(0)) /|(\operatorname{grad} f \mid \bar{d})(0)|^{3 / 2}$.

Proof. Let $\overline{d^{\varepsilon}}=\{X / \varepsilon \mid X \in \bar{d}\}$. Define the transformation $\mathcal{O}$ which maps $\bar{d}$ onto $\overline{d^{e}}$ by $\mathcal{Y}=\mathcal{Y}(X)=\left(x_{1} / \varepsilon, x_{2} / \varepsilon, 0\right)$. Define $s_{0}^{\varepsilon}=\left\{X \in \bar{d}|| X \mid=\varepsilon^{3 / 2}\right\}, s_{1}^{e}=$ $\left\{X \in \bar{d}|| X \mid=\varepsilon^{3 / 5}\right\}$ and $s_{2}^{e}=\left\{X \in \bar{d}|| X \mid=\varepsilon^{2 / 5}\right\}$. Define $s_{0}, s_{1}, s_{2}$ by $s_{i}=$ Y $\left(s_{i}^{e}\right), i=0,1,2$. Define $A_{0}^{e}$ to be the closed bounded subset of $\bar{d}$ with boundary $s_{0}^{\varepsilon}, A_{1}^{\varepsilon}$ the closed bounded subset of $\bar{d}$ with boundary $s_{0}^{\varepsilon} \cup s_{1}^{\varepsilon}, A_{2}^{\varepsilon}$ the closed bounded subset of $\bar{d}$ with boundary $s_{1}^{e} \cup s_{2}^{e}, A_{3}^{\varepsilon}=\bar{d}-\left(A_{0}^{\varepsilon} \cup A_{1}^{e} \cup A_{2}^{e}\right)$. Define 
$A_{i}=\mathcal{Y}\left(A_{i}^{e}\right)$. Note that the transformation $\mathcal{Y}$, the regions $s_{i}, A_{j}, i=0,1,2, j=$ $0,1,2,3$, depend on the parameter $\varepsilon$. However, the assertions of the theorem are only claimed to be true for each sufficiently small fixed positive $\varepsilon$.

Now compute $u_{e}(X) \mid \bar{d}$ in terms of the new coordinate $\mathcal{Y}$

$$
\begin{aligned}
u_{\varepsilon}(X) & =\varepsilon /\left(\varepsilon^{2} y_{1}^{2}+\varepsilon^{2} y_{2}^{2}+\varepsilon^{2}\right)^{1 / 2}=1 /\left(y_{1}^{2}+y_{2}^{2}+1\right)^{1 / 2} \\
& =1 /\left(1+|\mathcal{Y}|^{2}\right)^{1 / 2} .
\end{aligned}
$$

(46) demonstrates that $u_{\varepsilon}(X)$, when expressed in the $\mathcal{O}$ coordinates, is independent of the parameter $\varepsilon$. For convenience, define the function $u$ on $\overline{d^{e}}$ by

$$
u(\mathcal{Y})=1 /\left(1+|\mathcal{Y}|^{2}\right)^{1 / 2}
$$

Define the operators $\operatorname{grad}_{y}$ and $H_{y}$ as follows. Suppose $\psi$ is a function of class $C^{2}$ on $\overline{d^{e}}$; then $\operatorname{grad}_{y}(\psi)=\left(\partial \psi / \partial y_{1}, \partial \psi / \partial y_{2}\right) . H_{y} \psi$ is the $2 \times 2$ matrix $\left(\psi_{i j}\right), i, j=1,2$ such that $\psi_{i j}=\partial^{2} \psi / \partial y_{i} \partial y_{j}$. Define the operators $\operatorname{grad}_{2}$ and $\boldsymbol{H}_{2}$ on a function $\psi$ of class $C^{2}$ on $\bar{\Omega}$ by $\operatorname{grad}_{2} \psi=\left(\partial \psi / \partial x_{1}, \partial \psi / \partial x_{2}\right)$ and $H_{2} \psi=\left(\psi_{i j}\right), i, j=1,2$, with $\psi_{i j}=\partial^{2} \psi / \partial x_{i} \partial x_{j}$. Clearly the following operator identities hold from the definition of $\operatorname{grad}_{2}, \operatorname{grad}_{y}, H_{2}$ and $H_{y}$.

$$
\begin{gathered}
\operatorname{grad}_{y}=\varepsilon \operatorname{grad}_{2} . \\
H_{y}=\varepsilon^{2} H_{2} .
\end{gathered}
$$

Define the $C^{2}$ family $\mathcal{F}$ by $f_{\varepsilon}(\mathcal{Y})=f(\varepsilon \mathcal{Y})$. Define the $C^{2}$ family $\mathcal{T}$ of functions by $t_{e}(\mathcal{Y})=f_{\varepsilon}(\mathcal{Y})+u(\mathcal{Y})=v_{\varepsilon}(\varepsilon \mathcal{Y})$. Since for any fixed positive $\varepsilon$, the functions $v_{\varepsilon}$ and $t_{e}$ differ only by a dilation, any information about the critical points of $t_{\varepsilon}$ on $\frac{v_{e}}{d^{e}}$ may be interpreted as information concerning the critical points of $v_{e}$ on $\bar{d}$.

Let $W(\mathscr{Y})$ be a matrix-valued function on $\overline{d^{e}}$ defined by $W(\mathscr{Y})=\left(w_{i j}\right), i, j=$ 1,2 , where $w_{i j}=3 y_{i} y_{j} /|\mathcal{Y}|^{2}$ provided $|\mathcal{Y}| \neq 0$, and $w_{i j}(0)=0$. Let $I$ equal the $2 \times 2$ identity matrix. Define $\theta(\mathcal{Y})=|\mathcal{Y}|^{2}+\left(1+|\mathcal{Y}|^{2}\right)=1-\left(1 /\left(1+|\mathcal{Y}|^{2}\right)\right)$. From (48) and (49) it follows that

$$
\begin{gathered}
\operatorname{grad}_{y}(u)=-\mathcal{Y} /\left(1+|\mathcal{Y}|^{2}\right)^{3 / 2} \\
H_{y} u=(\theta(\mathcal{Y}) W(\mathcal{Y})-I) /\left(1+|\mathcal{Y}|^{2}\right)^{3 / 2}
\end{gathered}
$$

By hypothesis there exist numbers $0<a<A$ such that if $X \in \bar{d}$ then

$$
a<\left|\operatorname{grad}_{2}(f)\right|<A \text {. }
$$

(48), (50), and (52) clearly demonstrate that for sufficiently small positive $\varepsilon$, $\operatorname{grad}_{y}(u)$ dominates $\operatorname{grad}_{y}\left(f_{\varepsilon}\right)$ on the boundary of $A_{0}$. Therefore by (50), $\operatorname{grad}_{y}\left(t_{\varepsilon}\right) \cdot$ $\mathscr{Y} /|\mathcal{Y}|<0$ for each sufficiently small positive $\varepsilon$ and $\mathcal{Y} \in s_{0}$. In other words, all of the boundary of $A_{0}$ is entrant for the function $t_{\varepsilon}$ for sufficiently small positive $\varepsilon$. Let $\mu_{i}^{0}, i=0,1$ be the boundary type numbers of $t_{e}$ on $A_{0}$. Since $u$ is constant on $s_{0}$, it follows that $\mu_{i}^{0}, i=0,1$ are the type numbers of the restriction of $f_{e}$ to $s_{0}$ and are identical to the type numbers of $f \mid s_{0}^{e}$. We may compute the type numbers of $f \mid s_{0}^{e}$ for sufficiently small positive $\varepsilon$ using Corollary 3 of Lemma 1 and obtain

$$
\mu_{0}^{0}=\mu_{1}^{0}=1 \text {. }
$$


If $t_{\varepsilon}$ is nondegenerate on $A_{0}$, then let $m_{i}^{(0)}$ equal the interior type numbers of $t_{e}$ on $A_{0}$ for $i=0,1,2$. Then for sufficiently small positive $\varepsilon$ we have

$$
m_{0}^{(0)}+m_{2}^{(0)}-m_{1}^{(0)}=1 .
$$

It follows from the definition that norm $_{2}(W) \leqslant 3$, where norm ${ }_{2}$ is the customary 2-norm of the matrix. Clearly the function $\theta \rightarrow 0$ uniformly on $A_{0}$ as $\varepsilon \rightarrow 0$. In addition, $H_{y} f_{\varepsilon} \rightarrow 0$ uniformly on $\overline{d^{\varepsilon}}$ as $\varepsilon \rightarrow 0$. Therefore by (51), for each sufficiently small positive $\varepsilon$,

$$
\operatorname{index}\left(H_{y} t_{e}\right)=2 \text { if } y \in A_{0} .
$$

Therefore from (54) and (55) we have that for each sufficiently small positive $\varepsilon$, the function $t_{\varepsilon}$ has a unique critical point $p_{0}^{\varepsilon}$ on $A_{0}$ and $\operatorname{index}\left(p_{0}^{\varepsilon}\right)=2$, i.e.

$$
m_{2}^{(0)}=1, \quad m_{0}^{(0)}=m_{1}^{(0)}=0 .
$$

If $\mathcal{Y} \in A_{1}$, then the following inequality follows from the definition of the region $A_{1}$.

$$
\sqrt{\varepsilon} \leqslant|\mathcal{Y}| \leqslant \varepsilon^{-2 / 5} \text {. }
$$

It follows then from (48) and (50) that

$$
\left|\operatorname{grad}_{y}(u)\right|>\frac{1}{2} \cdot \varepsilon^{4 / 5} \text {. }
$$

(48) and (52) imply

$$
\operatorname{grad}_{y}\left(f_{\varepsilon}\right) \mid<A \cdot \varepsilon \quad \text { for all } y \in \overline{d^{\varepsilon}} .
$$

Therefore, for sufficiently small positive $\varepsilon$, the function $t_{\varepsilon}$ has no critical points on $A_{1}$.

By similar reasoning it follows from (48), (50) and (52) that for each $\mathcal{O}$ which belongs to $A_{3}$,

$$
\left|\operatorname{grad}_{y}\left(t_{\varepsilon}\right)\right| \geqslant\left|\operatorname{grad}_{y}\left(f_{\varepsilon}\right)\right|-\left|\operatorname{grad}_{y}(u)\right| \geqslant \varepsilon a-\varepsilon^{6 / 5}>0
$$

for sufficiently small positive $\varepsilon$. It is therefore apparent that for each sufficiently small positive $\varepsilon$, the functions $t_{e}$ can have no critical points on the region $A_{3}$.

Let $A^{e}=A_{0}^{e} \cup A_{1}^{e} \cup A_{2}^{e}$ and let $B=\left\{\mathcal{Y}(X) \mid X \in A^{e}\right\}$. Let $m_{0}, m_{1}, m_{2}$ equal the interior type numbers of $t_{\varepsilon}$ on $B$ and let $\mu_{0}, \mu_{1}$ equal the boundary type numbers of $t_{\varepsilon}$ on $B$. Then by previous arguments, for a fixed positive $\varepsilon, m_{0}, m_{1}, m_{2}$ are the interior type numbers of $v_{\varepsilon}$ on $A^{e}$ and $\mu_{0}, \mu_{1}$ are the boundary type numbers of $v_{\varepsilon}$ on $A^{e}$. On the other hand, the function $u_{e}$ is constant on $s_{2}^{e}$. Therefore the type numbers of $v_{e} \mid s_{2}^{e}$ equal the type numbers $f \mid s_{2}^{e}$. (60) assures that for each $\mathcal{Y}$ in $A_{3}$, in particular for each $\mathscr{Y}$ in the boundary of $A_{3}$, and sufficiently small positive $\varepsilon$, $\operatorname{grad}_{y}\left(f_{\varepsilon}\right)$ dominates $\operatorname{grad}_{y}(u)$. Certainly by (48) the same statement holds replacing $\operatorname{grad}_{y}\left(f_{\varepsilon}\right)$ with $\operatorname{grad}_{2}(f)$ and $\operatorname{grad}_{y}(u)$ with $\operatorname{grad}_{2}\left(u_{\varepsilon}\right)$. Therefore, for each sufficiently small positive $\varepsilon$, an entrant critical point of $v_{\varepsilon}$ on $s_{2}^{e}$ corresponds to an entrant critical point of $f$ on $s_{2}^{\varepsilon}$ and an emergent critical point of $v_{\varepsilon}$ on $s_{2}^{e}$ corresponds to an emergent critical point of $f$ on $s_{2}^{e}$. It then follows from Corollary 3 of Lemma 1 that, for each sufficiently small positive $\varepsilon$, the functions $f$ and $v_{\varepsilon}$ have exactly one entrant critical point on $s_{2}^{e}$ and this critical point is a minimum, i.e. 


$$
\mu_{0}=1, \quad \mu_{1}=0 .
$$

In the following paragraph let the index $i$ assume the values $0,1,2$. Let $m_{i}^{(2)}$ equal the interior type numbers of $t_{e}$ on $A_{2}$. Since it has been shown that for sufficiently small positive $\varepsilon, t_{\varepsilon}$ has no critical points on $A_{1}$, then we have, for sufficiently small positive $\varepsilon$,

$$
m_{i}=m_{i}^{(0)}+m_{i}^{(2)}
$$

From (56) and the fact that $B$ is a disk, it follows that for sufficiently small positive $\varepsilon$,

$$
\sum_{i=0}^{2}(-1)^{i} m_{i}^{(2)}=-1 .
$$

It will now be shown that for each sufficiently small positive $\varepsilon$ and $\mathcal{Y}$ in $A_{2}$, index $\left(H_{y} t_{e}\right)=1$. The definition of $t_{e},(49)$ and (41) imply

$$
\begin{aligned}
H_{y} t_{\varepsilon} & =H_{y}\left(u+f_{\varepsilon}\right)=H_{y} u+H_{y} f_{\varepsilon} \\
& =(\theta(\mathcal{Y}) W(\mathcal{Y})-I) /\left(1+|\mathcal{Y}|^{2}\right)^{3 / 2}+\varepsilon^{2} H_{2} f .
\end{aligned}
$$

The definition of the region $A_{2}$ implies that if $\mathscr{Y} \in A_{2}$,

$$
\varepsilon^{-2 / 5}<|\mathcal{Y}|<\varepsilon^{-3 / 5} \text {. }
$$

From (65), it follows immediately for $\varepsilon<1$,

$$
\frac{1}{2} \varepsilon^{9 / 5}<\left(1+|\mathcal{Y}|^{2}\right)^{-3 / 2}<\varepsilon^{6 / 5}
$$

Also from the definition of the function $\theta$ and (65), it follows that for $\mathcal{Y} \in A_{2}$,

$$
\lim _{\boldsymbol{e} \rightarrow 0^{+}} \theta(\mathcal{Y})=1 \text {. }
$$

From the definition of $W$ it is apparent that for $|\mathcal{Y}| \neq 0$, the eigenvalues of the matrix $W(\mathcal{Y})-I$ are 2 and -1 , independent of $\mathcal{Y}$. It now follows from (64), (65), and (67) that for $\mathcal{Y} \in A_{2}$,

$$
\lim _{e \rightarrow 0^{+}}\left(1+|\mathcal{Y}|^{2}\right)^{3 / 2} H_{y} t_{e}=W-I .
$$

Therefore, from the fact that the index of a matrix is invariant under scalar multiplication, index $\left(H_{y} t_{e}\right)=\operatorname{index}(W-I)=1$.

Therefore from (63) and (68), it follows that for sufficiently small positive $\varepsilon$,

$$
m_{0}^{(2)}=m_{2}^{(2)}=0, \quad m_{1}^{(2)}=1 .
$$

In other words, for sufficiently small positive $\varepsilon$, the function $t_{\varepsilon}$ has a unique critical point $p_{1}^{e}$ on the region $A_{2} ; p_{1}^{e}$ is nondegenerate and index $\left(p_{1}^{e}\right)=1$. This means that for each sufficiently small positive $\varepsilon$, the function $v_{e} \mid \bar{d}$ has exactly two critical points, $P_{0}^{e}$ and $P_{1}^{e}$, defined by $P_{0}^{e}=\mathcal{Y}^{-1}\left(p_{0}^{e}\right), P_{1}^{e}=\mathcal{Y}^{-1}\left(p_{1}^{e}\right)$ and $P_{0}^{e} \in A_{0}^{e}, P_{1}^{e} \in A_{2}^{e}$. This proves assertion 1 .

Assertion 2 is clear in light of the following equations.

$$
\begin{array}{cc}
\lim _{\varepsilon \rightarrow 0^{+}} \partial u_{\varepsilon}(X) / \partial x_{3}=0, & X \in A_{2}^{\varepsilon} . \\
\lim _{\varepsilon \rightarrow 0^{+}} \partial u_{\varepsilon}(X) / \partial x_{3}=+\infty, & X \in A_{0}^{\varepsilon} .
\end{array}
$$


The proof of assertion 3, from assertions 1,2 and the definition of the terms involved, is a routine calculation. This completes the proof.

TheOREM 5. Suppose the condition numbers $\mu_{0}, \mu_{1}, \mu_{2}, M_{1}, M_{2}, 1, R_{1}, R_{2}$ are harmonically realizable. Then the following sets of condition numbers are also harmonically realizable.

a. $\mu_{0}+1, \mu_{1}+1, \mu_{0}, M_{1}, M_{2}, 1, R_{1}, R_{2}$.
b. $\mu_{0}, \mu_{1}+1, \mu_{2}+1, M_{1}, M_{2}, 1, R_{1}, R_{2}$.
c. $\mu_{0}, \mu_{1}+1, \mu_{2}, M_{1}, M_{2}+1,1, R_{1}, R_{2}$.
d. $\mu_{0}+1, \mu_{1}, \mu_{2}, M_{1}+1, M_{2}, 1, R_{1}, R_{2}$.
e. $\mu_{0}, \mu_{1}, \mu_{2}+1, M_{1}, M_{2}, 1, R_{1}, R_{2}+1$.
f. $\mu_{0}, \mu_{1}, \mu_{2}, M_{1}, M_{2}+1,1, R_{1}, R_{2}+1$.

Proof. Parts a and b of Theorem 5 appear as Lemma 5.2 of [2].

Assume that $u$ and $\Omega$ is a harmonic realization of the type numbers $\mu_{0}, \mu_{1}, \mu_{2}, M_{1}$, $M_{2}, 1, R_{1}, R_{2}$. Define $\Sigma, \Sigma^{+}, \Sigma^{-}$from the pair $u$ and $\Omega$ as in the preliminaries. To prove c, let $P$ be any point in $\Sigma^{-}$such that $\operatorname{grad}(u \mid \Sigma)(P) \neq 0$. Now modify $\Omega$ such that there exists a neighborhood of $P, U$, in $\Sigma^{-}$which is contained in a 2-dimensional linear manifold. It is clear that such a modification can be accomplished leaving $\Sigma$ in class $C^{2}$ and the condition numbers $\mu_{0}, \mu_{1}, \mu_{2}, M_{1}, M_{2}, 1, R_{1}, R_{2}$, unaltered. By perhaps a rotation and translation, it is possible to assume that $P=$ origin and $N(P)=\langle 0,0,1\rangle$. Define the $C^{2}$ family of functions $\mathscr{Q}$ as in Theorem 4. Let $\mathfrak{V}=\mathscr{Q}+u$.

Obviously for any fixed positive $\varepsilon, v_{\varepsilon}$ is harmonic in a neighborhood of $\Omega$. Theorem 4 implies the existence of a positive number $\varepsilon_{0}$ such that for $0<\varepsilon<\varepsilon_{0}$, $v_{\varepsilon} \mid \bar{U}$ has exactly one entrant critical point; $P_{\varepsilon}$ is n.d. and the index of $P_{\varepsilon}$ is one. Lemma 1 and the fact that $u_{\varepsilon}$ and all its derivatives tend to 0 as $\varepsilon$ tends to 0 uniformly on any subset of $R^{3}$ which excludes a fixed neighborhood of the origin assure that outside any fixed neighborhood of the origin, and for sufficiently small positive $\varepsilon$, the critical points of $v_{\varepsilon}$ and $v_{\varepsilon} \mid \Sigma^{-}$correspond to the critical points of $u$ and $u \mid \Sigma^{-}$respectively as to number and index.

In addition, Theorem 3 asserts that for each sufficiently small positive $\varepsilon$, the function $v_{\varepsilon}$ has a critical point, $Q_{\varepsilon}=\left\langle q_{1_{\varepsilon}}, q_{2_{e}}, q_{3_{\varepsilon}}\right\rangle, Q_{\varepsilon}$ has index 2 and $Q_{\varepsilon}$ tends to 0 as $\varepsilon \rightarrow 0$. Moreover, since 0 is an ordinary point of $u$, there exists a fixed neighborhood $H$ of 0 in $R^{3}$ such that for each sufficiently small positive $\varepsilon, Q_{e}$ is the only critical point of $u_{\varepsilon}$ on $\bar{H}$. By the fact that $\operatorname{grad}(u(0)) \cdot N(0)<0$, and assertion 3 of Theorem 3, it follows that $q_{3}<\varepsilon-$ const $\varepsilon^{1 / 2}<0$ for all sufficiently small positive $\varepsilon$. Therefore, for sufficiently small positive $\varepsilon, Q_{\varepsilon}$ is contained in $\Omega$. From its definition, it follows that $u_{e}$ and all its derivatives tend to 0 uniformly on $R^{3}-H$. Therefore the addition of $u_{e}$ to the function $u$ adds one more critical point, $Q_{e}$, to the critical set of $u$ on $\Omega$. This statement combined with the proven values of the indices of $P_{\varepsilon}$ and $Q_{\varepsilon}$ complete the proof of part c.

To prove part d, choose $P$ to be a point in $\Sigma^{+}$and $v_{e}=u-u_{e}$. The proof now proceeds in a manner completely analogous to the proof of part $c$. 
To prove part e, pick a point $P$ in $\Omega$ such that $\operatorname{grad}(u(P)) \neq 0$. Let $\Omega_{e}^{\prime}=\{\Omega-$ (ball of radius $\varepsilon$ centered at $P$ ) $\}$. Clearly, for sufficiently small positive $\varepsilon$, the second betti number of $\Omega_{\varepsilon}^{\prime}=R_{2}+1$. By Corollary 3 of Lemma 2, for sufficiently small positive $\varepsilon$, the second boundary type number of $u$ on $\Omega_{\varepsilon}^{\prime}=\mu_{2}+1$. This proves part e.

To prove part $\mathrm{f}$, define $\Omega_{\varepsilon}^{\prime}$ as above. Define $u_{\varepsilon}^{\prime}$ on $\Omega_{\varepsilon}^{\prime}$ by $u_{\varepsilon}^{\prime}(X)=\varepsilon /|P-X|+u$, a harmonic function for each fixed $\varepsilon$.

$$
\operatorname{grad}\left(u_{\varepsilon}^{\prime}\right)=\operatorname{grad}(u)+\varepsilon \cdot(P-X) /|P-X|^{3}
$$

Clearly, from (72), for sufficiently small positive $\varepsilon$, the function $u_{\varepsilon}^{\prime}$ is emergent on the portion of boundary of $\Omega_{\varepsilon}^{\prime}$ which consists of sphere of radius $\varepsilon$ centered at $P$ \}. Therefore, for sufficiently small positive $\varepsilon$, the boundary type numbers of $u_{\varepsilon}^{\prime}$ on $\Omega_{\varepsilon}^{\prime}$ are identical to those of $u$ on $\Omega$. By Theorem 3 , the function $u_{e}^{\prime}$ has exactly one more critical point on $\Omega_{\varepsilon}^{\prime}$ than did $u$ on $\Omega$, and this critical point is n.d. with index 2. Lemma 1 assures that the critical points of $u_{e}^{\prime}$ outside any fixed neighborhood of $P$ correspond in number and in index to the critical points of $u$ on $\Omega$ for sufficiently small positive $\varepsilon$. This completes the proof of part $f$.

THEOREM 6. Suppose the condition numbers $\mu_{0}, \mu_{1}, \mu_{2}, M_{1}, M_{2}, 1, R_{1}, R_{2}$ are nonnegative and satisfy (1). Then this set of integers is harmonically realizable.

Proof. The strategy of this proof is to reduce the given set of condition numbers, by applying Theorem 5 , to a set of elementary condition numbers which have been proved harmonically realizable.

I. By iterated use of parts e and $\mathrm{f}$ of Theorem 5, it is possible to reduce the given set of type numbers to one of the following two forms.

a. $\mu_{0}^{\prime}, \mu_{1}^{\prime}, \mu_{2}^{\prime}, M_{1}^{\prime}, M_{2}^{\prime}, 1, R_{1}^{\prime}, 0$.

b. $\mu_{0}^{\prime}, \mu_{1}^{\prime}, 0, M_{1}^{\prime}, 0,1, R_{1}^{\prime}, R_{2}^{\prime}$.

The inequalities of (1) guarantee form a may always be achieved.

II. By iterated use of parts a and $\mathrm{d}$ of Theorem 5 , it is possible to reduce numbers of the form Ia to numbers of one of the following two forms.

a. $1, \mu_{1}^{\prime}, \mu_{2}^{\prime}, M_{1}^{\prime}, M_{2}^{\prime}, 1, R_{1}^{\prime}, 0$.

b. $\mu_{0}^{\prime}, 0, \mu_{2}^{\prime}, 0, M_{2}^{\prime}, 1, R_{1}^{\prime}, 0$.

The inequalities of (1) guarantee form a may always be achieved.

III. By iterated use of parts $b$ and $c$ of Theorem 5 , it is possible to reduce numbers of the form IIa to one of the following two forms.

a. $1, \mu_{1}^{\prime}, \mu_{2}^{\prime}, M_{1}^{\prime}, M_{2}^{\prime}, 1, R_{1}^{\prime}, 0$, for $\mu_{1}^{\prime} \leqslant R_{1}^{\prime}$.

b. $1, \mu_{1}^{\prime}, 0, M_{1}^{\prime}, 0,1, R_{1}^{\prime}, 0$.

The inequalities of (1) guarantee form a may always be achieved.

IIIa is a set of elementary condition numbers which are harmonically realizable by Theorem 2 . By reversing the reduction process, it is possible to augment the numbers of IIIa to the original set of condition numbers which must then also be harmonically realizable by Theorem 5. This completes the proof of Theorem 6. 


\section{REFERENCES}

1. Marston Morse, Topological methods in the study of functions of a complex variable, Princeton Univ. Press, Princeton, N. J., 1947.

2. Lquilibrium points of harmonic potentials, J. Analyse Math. 23 (1970), 281-296.

3. M. Morse and S. S. Cairns, Critical point theory in global analysis and differential topology: An introduction, Pure and Applied Math., Vol. 33, Academic Press, New York and London, 1969.

Departmant of Mathibmatics, University of Tennesseb, KnOXVIlle, Tennesseb 37916 